\title{
A rare case of metastatic proximal-type epithelioid sarcoma of the ischioanal fossa: case report and literature review
}

\begin{abstract}
Epithelioid sarcoma (ES) is a rare high-grade sarcoma subtype that constitutes less than $1 \%$ of soft tissue sarcomas (STS).There are two types: distal-type epithelioid sarcoma and proximal-type epithelioid sarcoma, based on anatomic location and the histopathological features. The clinical presentation of ES is varied and can lead to a delay in diagnosis. histopathology examination followed by immunohistochemistry will help to establish the diagnosis. The treatment of choice of Localized ES is a radical excision with microscopically radical margins and perioperative radiotherapy. systemic therapies are used in cases of locally advanced or metastatic ES. We describe a case of reoccurring proximal-type epithelioid sarcoma of the ischioanal fossa. A 56-year-old man operated two years ago for a epithelioid sarcoma of the ischioanal fossa. The patient presented with reoccurring mass at the same location, Magnetic resonance imaging (MRI) of the pelvis showed a mass of the ischioanal fossa. computed tomography (CT) of the chest, abdomen, and pelvis showed multiple pulmonary and liver metastasis. Histopathological features and immunohistochemistry were those of proximal type epithelioid sarcoma. He received intravenous doxorubicin with a partial response after 3 cycles of treatment.
\end{abstract}

Keywords: epithelioid sarcoma, soft tissue sarcomas, proximal type, radiotherapy, chemotherapy
Volume 12 Issue 3 - 202 I

\author{
Harrak Soukaina, Lemsanes Siham, Razine \\ Sawsan, Benchekroun Khadija, Lkhouyaali \\ Siham, Abahsaine Halima, Boutayeb Saber, \\ Errihani Hassan \\ Department of Medical Oncology, National Institute of \\ Oncology, Morocco
}

Correspondence: Harrak Soukaina, Department of Medical Oncology, National Institute of Oncology, 10100, Rabat, Morocco,Tel 002I2624459709, Email Soukaina.hrk@gmail.com

Received: June 20, 202I | Published: July 01, 2021

\section{Introduction}

Epithelioid sarcoma (ES) is a high grade malignant mesenchyma tumor that exhibits epithelioid cytomorphology and a predominantly epithelial phenotype. It was first described in 1970 by Franz Enzinger. ES is a soft tissue sarcomas (STS) subtype that is recognized in less than $1 \%$ of STS patients. ${ }^{2}$ It mostly occurs in the deep dermal or subcutaneous areas of the distal portions of the extremities of young adults particularly the hand, yet it can arise in any part of the body, including the penis, vulva, and perineum. ${ }^{3,4}$ The tumor occurs in all ages, however, it is most prevalent in individuals aged 20-40 years and is rarely found in children and older individuals. ${ }^{3}$ The clinical presentation of ES is usually non specific, it is frequently mis diagnosed as a benign process and this remains a challenge in terms of diagnosis and management. Surgical resection of localized disease has been the mainstay of care with an undefined role for pre or post-operative radiation therapy. ${ }^{5}$ Systemic therapy in the metastatic setting with either doxorubicin or gemcitabine based therapies are often given, however, outcomes are poor with a short duration of response. ${ }^{6,7}$ Recently tazemetostat demonstrated significant activity in ES. The prognosis of such a disease is dismal with high mortality rates in the recurrent and metastatic cases. ${ }^{8}$ In this report, we describe a case of an 56-year-old men with a metastatic proximal epithelioid sarcoma of the ischioanal fossa treated by doxorubicin.

\section{Case report}

A 56-year-old man with any medical history was referred to our institution for evaluation of reoccurring mass on the ischioanal fossa. The patient reported a left perianal mass measured to about $5 \mathrm{~cm}, 6$ months duration before his first consultation. The patient has already had the mass surgically removed. The basis of the histopathological examination and immunohistochemistry finding, the patient was diagnosed as having a proximal type localized epithelioid sarcoma of the ischioanal fossa. No adjuvant chemotherapy or radiation was recommended. The patient did not attend his schedule followup meetings at the hospital. Two years after the surgery, he noticed recurrence of the mass at the same location.

Follow this finding he was referred to our institution for the management. On examination, a a left perianal mass was noted measured to about $4 \mathrm{~cm}$, there were no palpable lymph nodes. The rest of the physical exam was unremarkable. A magnetic resonance imaging (MRI) of the pelvis was performed and showed a $54 \times 44 \times 72 \mathrm{~mm}$ mass of the left ischioanal fossa, infiltrating the levator ani muscle homolaterally without endopelvic extension (Figure 1). CT showed liver and lung metastasis.

The patient had a biopsy of the mass whose Histological examination revealed multinodular pleomorphic proliferation of epithelioid cells with abundant eosinophilic cytoplasm, The tumor cell nuclei were round or oval, hyperchromatic and presenting a low number of mitoses. Immunostaining showed positivity for CD34, epithelial membrane antigen (EMA), and pan-cytokeratin, and negativity for PS100, myogenin and INI1. Ki 67 was $10 \%$. Because of the presence of multiple metastasis, the patient was therefore recommended to undergo chemotherapy, he received first line palliative chemotherapy with doxorubicin $75 \mathrm{mg}$ intravenously every 3 weeks.

Reassessment CT scan performed after three cycles of chemotherapy showed a partial response. He has now been treated with 5 cycles of doxorubicin and the chemotherapy cycles were exceedingly well tolerated by the patient. A second evaluation scan will be scheduled after the sixth cycle. 


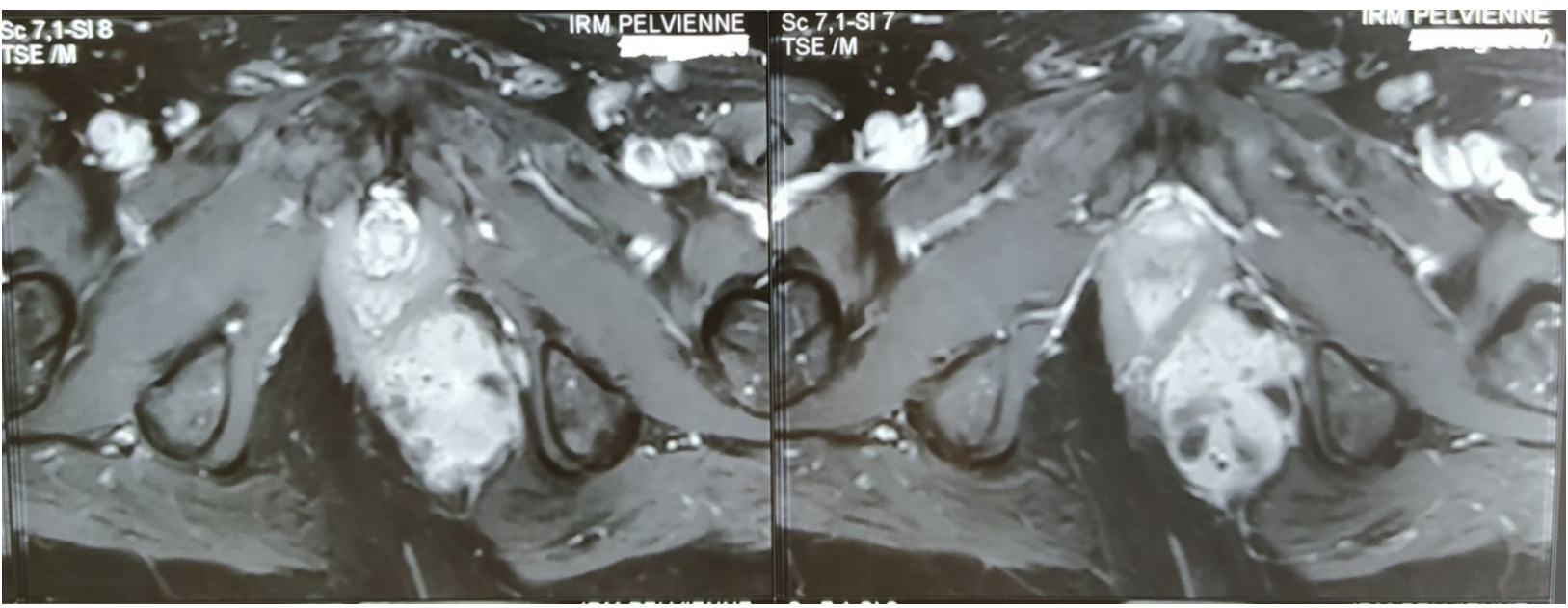

Figure I Pelvis MRI axial showing a mass of the left ischioanal fossa, which was measured at about $54 \times 44 \times 72 \mathrm{~mm}$.

\section{Discussion}

Epithelioid sarcoma (ES) is a rare mesenchymal tumor that occurs mostly in the dermal or subcutaneous area of the distal extremities. Generally, ES is considered as a high-grade soft tissue sarcoma.9 ES occurs in less than $1 \%$ of all adult soft-tissue sarcomas and approximately $4 \%-8 \%$ of pediatric non-rhabdomyosarcomatous sarcoma. ${ }^{10,11}$ Traditionally, ES is divided in two subtypes: The classic form (distal-type) and the proximal-type. The classic form is seen more in teenagers and young adults. The proximal-type ES is a rarer form, which mainly affects adults and is often presented as a more aggressive and mainly affects adults..$^{12}$ Distal-type ES is characterized as afirm, non-tender, slow-growing tumor with predilection towards distal extremities, especially the hands. ${ }^{13}$ Different from distal-type, proximal-type ES is characterized by deeper masses infiltrating soft-tissue, commonly with hemorrhage and necrosis, affecting axial proximal regions. ${ }^{12}$ Reported sites include the pelvis and perineal region, pubic region, and vulva, buttock, hip, penis, axilla, mediastinum, occiput, and forearm. ${ }^{12-14}$

Due to the clinical presentation of ES is usually non specific, the painless and indolent nature of the tumour, and it may initially look like benign lesion, ES is frequently mis diagnosed as a benign process and the diagnosis is not made until the tumor has already reached an advanced stage. As it is difficult to diagnose ES clinically, the biopsy with immunohistochemistry plays an important role in confirming the diagnosis. Classic distal-type ES shows characteristic histological features of a nodular growth pattern of plump epithelioid cells with a relatively abundant eosinophilic cytoplasm. Spindled cells can also be seen. The nodules typically contain prominent central necrosis. On the other hand, proximal-type ES is characterized by multinodular distributions and sheets of large polygonal cells with pleomorphic vesicular nuclei and prominent nucleoli. Rhabdoid features are also frequently observed in this form of ES. The nodules sometimes contain central necrosis, but this may be with or without the geographic pattern of necrosis typical of classic ES. ${ }^{15,16}$ The histologic type in our case was similar to the proximal type despite the central location of the tumor.

Immunohistochemical staining usually reveals cytoplasmic immunoreactivity for cytokeratin, vimentin, and EMA, where as S-100 and CD31 staining are usually negative. Epithelioid sarcoma commonly displays membranous positivity for EMA, in contrast to other majority of soft tissue sarcomas, which show nonspecific cytoplasmic EMA expression. About $50 \%$ of the cases express CD $34 .{ }^{14}$ Loss of integrase interactor 1 (INI1) function is the most common alteration found in ES, occurring in nearly $90 \%$ of cases. ${ }^{17}$ This loss of INI-1 protein is related to biallelic inactivation of the tumor suppressor gene SMARCB1/ INI1, which is located at chromosome $22 q 11.2 .{ }^{17} \mathrm{ES}$ has an unfavourable prognosis with reported $77 \%$ local recurrence and $45 \%$ distant metastasis rates, usually to regional lymph nodes, lungs, skin, scalp, bone, brain and other soft tissue parts. ${ }^{18-20}$ Factors that are associated with a worse prognosis for ES are proximal and deep location, rhabdoid features, large size, older age, male sex, necrosis, and vascular invasion. ${ }^{15}$

Due to their rare incidence and the absence of dedicated clinical trials, specific recommendations and guidelines on the optimal management of ES are almost inexistent. First, complete surgical resection with optimal margins (R0) remains the mainstay of curative therapy in nonmetastatic, localized ES patients. ${ }^{4}$ For localized disease, neoadjuvant or adjuvant radiation therapy is often given to reduce local relapse ${ }^{21,22}$ but the role of adjuvant chemotherapy is unclear. ${ }^{14,23-25}$ Chemotherapy is widely used in locally advanced or metastatic ES, as in our patient with liver metastasis. The most commonly administered chemotherapy regimens are single-agent anthracycline therapy or the combination of an anthracycline with ifosfamide. ${ }^{6}$ A single group reported activity of a regimen combining gemcitabine with docetaxel, but the experience is limited to a small number of patients. ${ }^{7}$ Progression free survivals (PFS) reported in the literature are relatively low, with one study reporting a median PFS for gemcitabine-based regimens of 4 months and doxorubicin regimens of 6 months. ${ }^{26}$

Many expectations arose around targeted therapies for ES. Signs of activity in a few cases have also been reported with pazopanib, the only anti-angiogenic compound currently licenced in advanced, pretreated, nonadipocytic, STSs and trabectedin. ${ }^{27,28}$ very limited data on the activity of immunotherapy in ES are currently available, with one response ascribed to pembrolizumab. ${ }^{29}$ Recently, the Food and Drug Administration (FDA) has approved tazemetostat, an EZH2 inhibitor that has the potential to block this genetic alteration, demonstrated significant activity in several hematological and solid tumors, for the treatment of adults and pediatric patients aged 16 years and older with metastatic or locally advanced epithelioid sarcoma not eligible for radical resection. The approval has been granted based on the results of cohort 5 of the phase 2 clinical trial (NCT02601950). ${ }^{30,31}$ 


\section{Conclusion}

Epithelioid sarcoma (ES) is a rare mesenchymal tumor, This tumor has a high risk for local recurrence and distant metastasis. Prognosis, as with most malignancies, is primarily determined by the clinical stage of the disease. Complete resection while is considered as the main treatment. Tazemetostat, has shown promising results in ES patients. Novel therapies, including immunotherapy, are still needed.

\section{Acknowledgments}

None.

\section{Conflicts of interest}

The authors declare that they have no conflict of interests.

\section{References}

1. Enzinger FM. Epitheloid sarcoma. A sarcoma simulating a granuloma or a carcinoma. Cancer. 1970;26(5):1029-1041.

2. McPhee M, McGrath BE, Zhang P, et al. Soft tissue sarcoma of the hand $J$ Hand Surg. 1999;24(5):1001-1007.

3. Rekhi B, Gorad BD, Chinoy RF. Proximal-type epithelioid sarcoma-a rare, aggressive subtype of epithelioid sarcoma presenting as a recurrent perineal mass in a middle-aged male. World J Surg Oncol. 2007;5:28.

4. de Visscher SA, van Ginkel RJ, Wobbes T, et al. Epithelioid sarcoma: Still an only surgically curable disease. Cancer. 2006;107(3):606-612.

5. Chase DR, Enzinger FM. Epithelioid sarcoma: Diagnosis, prognostic indicators, and treatment. Am J Surg Pathol. 1985;9(4):241-263.

6. Jones RL, Constantinidou A, Olmos D, et al. Role of palliative chemotherapy in advanced epithelioid sarcoma. Am J Clin Oncol. 2012;35(4):351-357.

7. Pink D, Richter S, Gerdes S, et al. Gemcitabine and docetaxel for epithelioid sarcoma: results from a retrospective, multi-institutional analysis. Oncology. 2014;87(2):95-103.

8. Peacock ZS, Lam DK, Cox DP, et al. Metastatic epithelioid angiosarcoma to the mandible: report of a case and review of the literature. Int J Oral Maxillofac Surg. 2013;42(6):702-706.

9. Jeon SY, Yhim HY, Lee NR. Epithelioid sarcoma with spontaneouspneumothorax and massive pleural effusion. Korean J Intern Med. 2016;31(1):191-193.

10. Jawad MU, Extein J, Min ES, et al. Prognostic factors for survival inpatients with epithelioid sarcoma: 441 cases from the SEER database. Clin Orthop Relat Res. 2009;467(11):2939-2948.

11. Casanova M, Ferrari A, Collini P, et al. Epithelioid sarcoma in children and adolescents: a report from the Italian Softtissue Sarcoma Committee. Cancer. 2006;106(3):708-717.

12. Guillou L, Wadden C, Coindre JM, et al. «Proximal-type» epithelioid sarcoma, a distinctive aggressive neoplasm showing rhabdoid features. Clinicopathologic, immunohistochemical, and ultrastructural study of a series. Am J Surg Pathol. 1997;21(2):130-146.

13. De Visscher SAHJ, Van Ginkel RJ, Wobbes T, et al. Epithelioid sarcoma: still an only surgically curable disease. Cancer. 2006;107(3):606-612.

14. Hasegawa T, Matsuno Y, Shimoda T, et al. Proximal-type epithelioid sarcoma: a clinicopathologic study of 20 cases. Mod Pathol. 2001;14(7):655-663.
15. Thway K, Jones RL, Noujaim J, et al. Epithelioid sarcoma: diagnostic features and genetics. Adv Anat Pathol. 2016;23(1):41-49.

16. Li Y, Cao G, Tao X, et al. Clinicopathologic features of epithelioid sarcoma: report of seventeen cases and review of literature. Int J Clin Exp Pathol. 2019;12(8):3042-3048.

17. Hornick JL, Dal Cin P, Fletcher CDM. Loss of INI1 Expression is Characteristic of Both Conventional and Proximal-type Epithelioid Sarcoma. Am J Surg Pathol. 2009;33(4):542-550.

18. Gurwale SG, Gore CR, Kaur S, et al. Primary epithelioid sarcoma of scalp. Asian Journal of Oncology. 2017;3(1):71-73.

19. Chbani L, Guillou L, Terrier P, et al. Epithelioid SarcomaA Clinicopathologic and Immunohistochemical Analysis of 106 Cases From the French Sarcoma Group. American Journal of Clinical Pathology. 2009;131(2):222-227.

20. Kaya EA, Broadbent TJ, Thomas CJ et al. Primary Epithelioid Sarcoma of Orbit: A Case Report and Review of the Literature. Case Reports in Oncological Medicine. 2018;2018:398716.

21. Shimm DS, Suit HD. Radiation therapy of epithelioid sarcoma. Cancer. 1983;52(6):1022-1025.

22. Livi L, Shah N, Paiar F, et al. Treatment of epithelioid sarcoma at the Royal Marsden Hospital. Sarcoma. 2003;7(3-4):149-152.

23. Baratti D, Pennacchioli E, Casali PG, et al. Epithelioid sarcoma: prognostic factors and survival in a series of patients treated at a single institution. Ann Surg Oncol. 2007;14(12):3542-3551.

24. Wolf PS, Flum DR, Tanas MR, et al. Epithelioid sarcoma: the University of Washington experience. Am J Surg. 2008;196(3):407-412.

25. Callister MD, Ballo MT, Pisters PW, et al. Epithelioid sarcoma: results of conservative surgery and radiotherapy. Int J Radiat Oncol Biol Phys. 2001;51(2):384-391.

26. Frezza AM, Jones RL, Lo Vullo S, et al. Anthracycline, Gemcitabine, and Pazopanib in Epithelioid Sarcoma: A Multi-institutional Case Series. JAMA Oncol. 2018;4(9):e180219.

27. Irimura S, Nishimoto K, Kikuta $K$, et al. Successful treatment with pazopanib for multiple lung metastases of inguinal epithelioid sarcoma: a case report. Case Rep Oncol. 2015;8(3):378-384.

28. Nakamura T, Matsumine A, Kawai A, et al. The clinical outcome of pazopanib treatment in Japanese patients with relapsed soft tissue sarcoma: a Japanese Musculoskeletal Oncology Group (JMOG) study. Cancer. 2016;122(9):1408-1416.

29. Geoerger B, Kang HJ, Yalon-Oren M, et al. Pembrolizumab in paediatric patients with advanced melanoma or a PD-L1-positive, advanced, relapsed, or refractory solid tumour or lymphoma (KEYNOTE-051): interim analysis of an open-label, single-arm, Phase I-II trial. Lancet Oncol. 2020;21(1):121-133.

30. Italiano A, Soria JC, Toulmonde M, et al. Tazemetostat, an EZH2 inhibitor, in relapsed or refractory B-cell nonHodgkin lymphoma andadvanced solid tumours: a first-in-human, open-label, Phase I study. Lancet Oncol. 2018;19(5): 649-659.

31. Stacchiotti S, Schoski P, Jones R, et al. Safety and efficacy of tazemetostat, a first-in-class EZH2 inhibitor, in patients (pts) with epithelioid sarcoma (ES) (NCT02601950). J Clin Oncol. 2019;37(15):11003. 